\title{
ON MANIFOLDS WITH INVOLUTION
}

\author{
BY J. M. BOARDMAN
}

Communicated by P. E. Conner, July 25, 1966

We consider a smooth involution $\omega$ on a smooth closed $n$-manifold $M$ from the bordism point of view, as in [2, Chapter IV]. We know that the fixed-point set of $\omega$ is the disjoint union of submanifolds; let $k$ be the maximum dimension of these. It is clear that if $\omega$ is free, $M$ bounds a 1-disk bundle over the orbit space. Now fix $k$, and let $n$, $M$, and $\omega$ vary. Conner and Floyd prove [2, Theorem (27.1)] that if $M$ does not bound, $n$ cannot be arbitrarily large. Their proof is nonconstructive, and fails to give an upper bound for $n$. We obtain the precise bound.

Theorem 1. Suppose $k$ is the maximum dimension of the fixed-point submanifolds of the smooth involution $\omega$ on the closed nonbounding $n$ manifold $M$. Then $n \leqq 5 k / 2$ (if $k$ is even) or $n \leqq(5 k-1) / 2$ (if $k$ is odd). Further, if we are given that the unoriented cobordism class of $M$ is indecomposable, then $n \leqq 2 k+1$.

Examples in the extremal dimensions are easily constructed. Take homogeneous coordinates $\left(x_{0}, x_{1}, x_{2}, \cdots, x_{i}, x_{1}^{\prime}, x_{2}^{\prime}, \cdots, x_{i}^{\prime}\right)$ on real projective $2 i$-space $P_{2 i}(i>0)$, and define the involution $\omega_{i}$ by

$$
\omega_{i}\left(x_{0}, x_{1}, \cdots, x_{i}, x_{1}^{\prime}, \cdots, x_{i}^{\prime}\right)=\left(x_{0}, x_{1}, \cdots, x_{i},-x_{1}^{\prime}, \cdots,-x_{i}^{\prime}\right) \text {. }
$$

Then the product involution $\omega_{i} \times \omega_{j}$ on $P_{2 i} \times P_{2 j}$ maps the hypersurface $H_{2 i, 2 j}$ defined by the equation

$$
x_{0} y_{0}+x_{1} y_{1}+\cdots+x_{i} y_{i}+x_{1}^{\prime} y_{1}^{\prime}+\cdots+x_{i}^{\prime} y_{i}^{\prime}=0
$$

into itself, where for clarity we take coordinates $\left(y_{0}, y_{1}, \cdots, y_{j}\right.$, $\left.y_{1}^{\prime}, \cdots, y_{j}^{\prime}\right)$ on $P_{2 j}$, and assume $i \leqq j$. The fixed-point dimension of $\omega_{i} \times \omega_{j} \mid H_{2 i, 2 j}$ is found to be $i+j-1$. The manifold $H_{2 i, 2 j}$ has dimension $2 i+2 j-1$ and its cobordism class $\left[H_{2 i, 2 j}\right]_{2}$ is indecomposable if and only if the binomial coefficient

$$
\left(\begin{array}{c}
i+j \\
i
\end{array}\right)
$$

is odd. We can always choose $i$ and $j$ satisfying this condition and $i+j=m$ whenever $m$ is not a power of 2 . As an example for the first assertion of Theorem 1, we take the product of many copies of the 5dimensional example $\left(H_{2,4}, \omega_{1} \times \omega_{2} \mid H_{2,4}\right)$, with possibly one copy of $\left(P_{2}, \omega_{1}\right)$. 
We deduce Theorem 1 from Theorems 2 and 3 below, which are purely algebraic. They concern the bordism $J$-homomorphism

$$
J_{m}: \mathfrak{N}_{i}(B O(m)) \rightarrow \mathfrak{N}_{i+m-1}(B O(1))
$$

defined in $[2, \S 25]$. (It has only a tenuous connection with the HopfWhitehead $J$-homomorphism, which could be written

$$
J: \pi_{i}(B O(m)) \rightarrow \pi_{i+m-1}\left(P_{m}\right) \text {.) }
$$

Let us recall from [2] the bordism classification of manifolds with involution $(M, \omega)$. The normal bundle in $M$ of the $i$-dimensional fixed-point set of $\omega$ determines an element $\nu_{i} \in \mathfrak{N}_{i}(B O(n-i))$. The main structure theorem (28.1) asserts that these elements characterize the bordism class of $(M, \omega)$, and are arbitrary, subject only to the condition

$$
\sum_{i} J_{n-i} \nu_{i}=0
$$

We stabilize $J_{n}$ by defining a homomorphism

$$
J: \mathfrak{N}_{*}(B O) \rightarrow \mathfrak{N}[[\theta]],
$$

where $\mathfrak{R}[[\theta]]$ is the ring of homogeneous formal power series over $\mathfrak{N}$ in an indeterminate $\theta$ of degree -1 . Given $\alpha \in \mathfrak{N}_{i}(B O)$, we put $J \alpha=\sum \alpha_{r} \theta^{r}$. To define the coefficient $\alpha_{r} \in \mathfrak{N}_{r}$, we first lift $\alpha$ to $\alpha^{\prime} \in \mathfrak{M}_{i}(B O(m))$ for some $m \geqq r-i+1$, and put $\alpha_{r}=\epsilon \Delta^{i+m-r-1} J_{m} \alpha^{\prime}$, where $\Delta$ is the bordism Smith homomorphism defined in [2, §26], and $\epsilon: \mathfrak{N}_{*}(B O(1)) \rightarrow \mathfrak{N}$ is the canonical augmentation; $\alpha_{r}$ is independent of $m$ by Theorem (26.4) of [2]. It is more natural to define $J: F \rightarrow \mathfrak{N}[[\theta]]$, where $F=\oplus_{i} \Re_{i}(B O)$, by extending linearly. Then the elements $\nu_{i}$, when included in $\mathfrak{N}_{*}(B O)$, may be added in $F$ to form an element $\nu \in F$. The relation $\sum_{i} J_{n-i} \nu_{i}=0$, and also (24.2) of [2], are combined in the following formula.

Theorem 2. $J \nu=[M]_{2} \theta^{n}+$ terms with higher powers of $\theta$.

Now the cross product of vector bundles makes $F$ into an ungraded polynomial ring. It is easy to see that

$$
J 1=1+\left[P_{2}\right]_{2} \theta^{2}+\left[P_{4}\right]_{2} \theta^{4}+\left[P_{6}\right]_{2} \theta^{6}+\cdots .
$$

Therefore we define $J^{\prime}: F \rightarrow \mathfrak{N}[[\theta]]$ by setting $J^{\prime} \alpha=(J \alpha) \cdot(J 1)^{-1}$, so that $J^{\prime} 1=1$. We may clearly replace $J$ by $J^{\prime}$ in Theorem 2 . We are interested in the case when

$$
\nu \in F_{k}=\oplus_{i=0}^{i=k} \Re_{i}(B O) \subset F .
$$


THEOREM 3. $J^{\prime}: F \rightarrow \mathfrak{N}[[\theta]]$ is a ring homomorphism. Further, we can find systems of polynomial generators $z_{i} \in \mathfrak{R}_{i}$ for $\mathfrak{N}$ and $x_{i}$ for $F$, for each $i$ not of the form $2^{q}-1$, such that

(a) $J^{\prime} x_{i}=z_{i} \theta^{i}+$ terms with higher powers of $\theta$,

(b) If we assign to $x_{i}$ the weight $i / 2$ ( $i$ even) or $(i-1) / 2$ ( $i$ odd), then $F_{k}$ consists of all polynomials of weight $\leqq k$ in the elements $x_{i}$.

There ought to be a direct geometric proof that $J^{\prime}$ is a ring homomorphism.

The computation of $J_{n}$, and hence of $J^{\prime}$, is in principle known from $[2$, Chapter IV]. All that is lacking is a certain amount of technique. Full details will appear in [1].

\section{REFERENCES}

1. J. M. Boardman, Unoriented bordism and cobordism, (to appear).

2. P. E. Conner and E. E. Floyd, Differentiable periodic maps, Springer-Verlag, Berlin, 1964.

UNIVERSITY OF WARWICK,

Coventry, ENGLAND 